\title{
Inflammatory pathways in the pathogenesis of iatrogenic laryngotracheal stenosis: what do we know?
}

\author{
Ruth J. Davis, Alexander T. Hillel \\ Department of Otolaryngology, Head and Neck Surgery, Johns Hopkins University School of Medicine, Baltimore, MD, USA \\ Contributions: (I) Conception and design: All authors; (II) Administrative support: None; (III) Provision of study materials or patients: None; (IV) \\ Collection and assembly of data: RJ Davis; (V) Data analysis and interpretation: None; (VI) Manuscript writing: All authors; (VII) Final approval of \\ manuscript: All authors. \\ Correspondence to: Alexander T. Hillel, MD. Department of Otolaryngology, Head and Neck Surgery, Johns Hopkins University, 601 North Caroline \\ Street, Baltimore, MD 21287, USA. Email: ahillel@jhmi.edu.
}

\begin{abstract}
Iatrogenic laryngotracheal stenosis (LTS) is pathologic airway narrowing due to scar formation following endotracheal intubation or tracheostomy. It is a chronic and recurrent disease that results in significant morbidity or even mortality. Current data suggests that inflammatory pathways play a significant role in mediating the deposition of collagen and extracellular matrix to generate this scar. In particular, the T-helper 2 cell and M2 macrophage axis appears to be activated in both animal models and patients with iatrogenic LTS. Interleukin-6 production in response to hypoxia has also been implicated. In this paper, we review the data supporting the roles of various inflammatory pathways in the pathogenesis of iatrogenic LTS, discuss potential therapeutic approaches targeting these mechanisms, and outline areas for future study.
\end{abstract}

Keywords: Tracheal stenosis; inflammation; helper T-cells; macrophages; interleukin-4

Submitted Jul 06, 2019. Accepted for publication Jan 02, 2020.

doi: $10.21037 /$ tcr.2020.01.21

View this article at: http://dx.doi.org/10.21037/tcr.2020.01.21

\section{Introduction}

Laryngotracheal stenosis (LTS) is a fibrotic disease leading to pathologic narrowing of the larynx, subglottis, and trachea. Causes of LTS include intubation injury (iatrogenic), radiation, autoimmune disease, or it may be idiopathic in nature (1). Iatrogenic LTS (iLTS) is the most common form, which occurs in $4-13 \%$ of adults and up to $11 \%$ of infants following prolonged intubation, or can occur following tracheostomy $(2,3)$. The consequences of LTS in its early stages include dysphonia and communication difficulties related to the turbulent airflow through areas of narrowing. Without intervention, LTS can develop into a life-threatening condition leading to progressive dyspnea and ultimately airway compromise (4).

Current management of LTS is primarily surgical, including serial dilations, tracheal resection, or permanent tracheostomy. Patients with iLTS have been found to have higher rates of tracheostomy dependence of up to
$66 \%$ compared to patients with idiopathic or traumatic etiologies (2). This demonstrates the heterogeneous nature of LTS of various etiologies, highlighting the particularly poor prognosis associated with iLTS and the limitations of current therapies. Treatment of iLTS is associated with significant health care expenditures of over $\$ 5,000$ per patient annually which is comparable to other chronic diseases including chronic obstructive pulmonary disease or diabetes mellitus (5). Medical alternatives or adjuncts to the surgical management of LTS are limited by a lack of mechanistic understanding of the pathogenesis of LTS. Currently utilized adjuncts include systemic or local steroid treatments, mitomycin $\mathrm{C}$ injections, antibiotics and other anti-inflammatory medications. However, evidence for each of these approaches is inconsistent, highlighting the need for a deeper understanding of the inflammatory mechanisms that contribute to fibrosis in order to provide targeted and effective treatment options $(6,7)$.

Pathologic specimens from iLTS patients demonstrate 
A
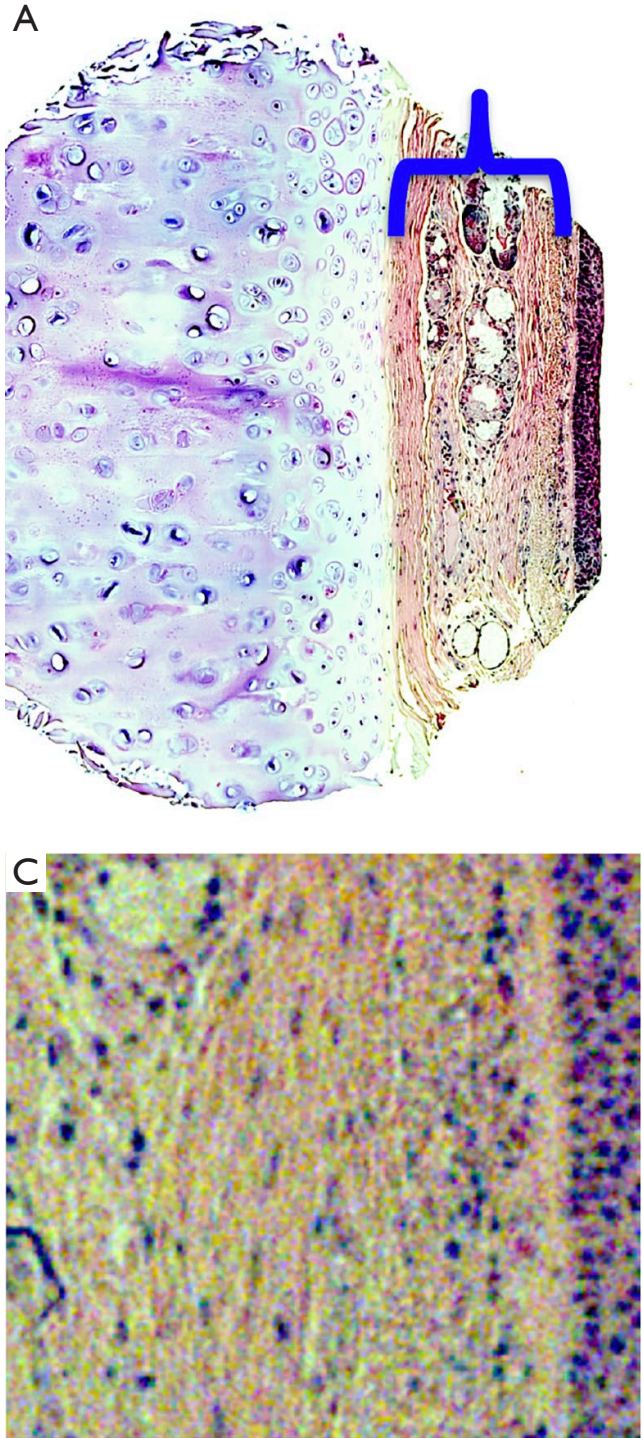

B
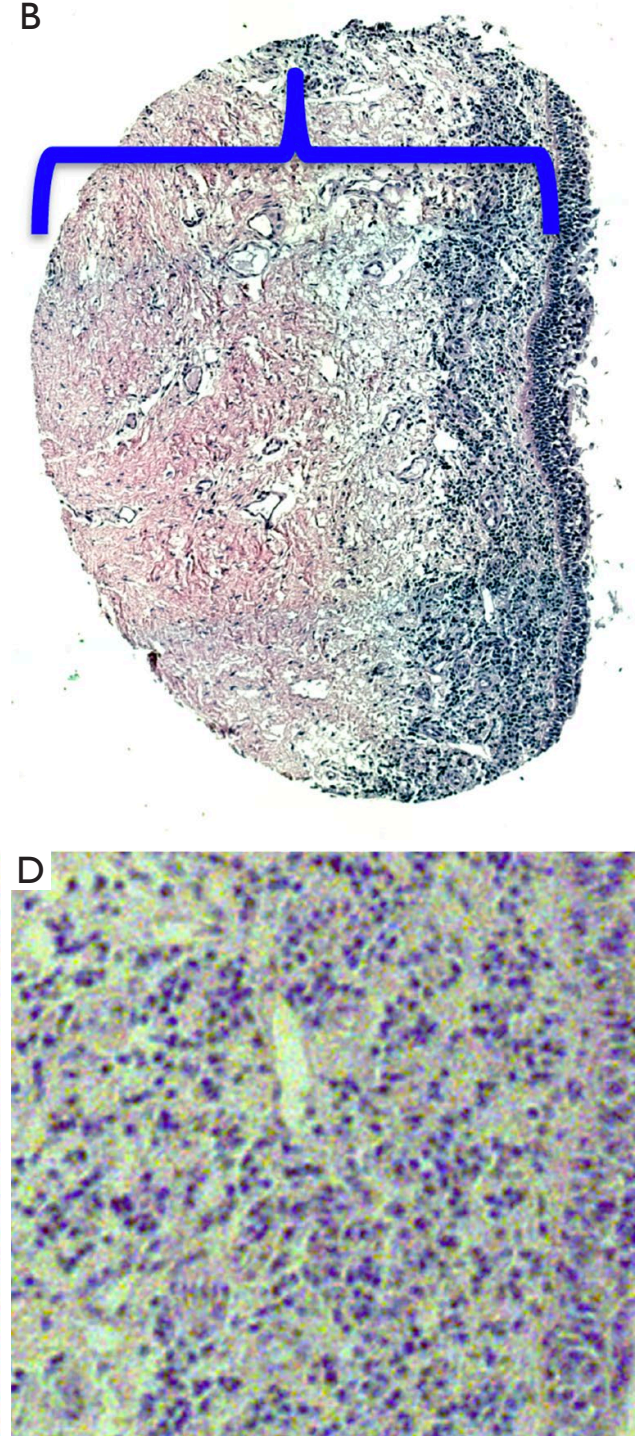

Figure 1 Histologic images of periepithelial specimens from normal and iLTS human tracheas. (A) Photomicrograph of a healthy control trachea demonstrating a normal lamina propria width (blue brackets, between the epithelium and underlying tracheal cartilage, $\times 50$ magnification) compared with (B) a specimen from a patient with iLTS demonstrating thickened fibrotic lamina propria and dense immune cell infiltrate just beneath irregular epithelium ( $\times 50$ magnification). Higher magnifications $(\times 400)$ of $(C)$ normal and $(D)$ diseased mucosa. All panels from hematoxylin and eosin-stained specimens. Figure and legend reused with permission from ref. (9).

thickening of the lamina propria with excessive collagen and extracellular matrix (ECM) that leads to pathologic narrowing of the tracheal lumen $(8,9)$ (Figure 1). Historically, this fibrotic response to airway injury was thought to result in the development of inert scar, a fixed end point that simply needed to be removed in order to obtain symptomatic improvement. However, beginning in the late 1980 s, researchers began to investigate the cellular basis of laryngotracheal mucosal wound healing. Minnigerode and Richter proposed mechanical injury resulted in epithelial defects and increased local bacterial contamination which in turn propagated chronic inflammation in the mucosa (8). More recent research has confirmed that theory as the paradigm shifted towards viewing LTS as an active fibroinflammatory process mediated by aberrant functioning of the body's own 
immune cells in response to injury (1,9-12).

In physiologic wound healing, injured epithelial and endothelial cells release growth factors and chemokines that trigger clot formation and recruit leukocytes to the site of injury (13). These leukocytes then release pro-inflammatory cytokines that recruit and activate fibroblasts. Specifically, macrophages and neutrophils eliminate dead cells and tissue debris and produce cytokines and chemokines to recruit and activate T-cells. These T-cells produce pro-inflammatory cytokines such as IL-13 that recruit and activate fibroblasts. In concert with neovascularization and wound contraction, fibroblast deposition of collagen and fibronectin is balanced by extracellular matrix remodeling by matrix metalloproteinases (MMPs). When these functions occur in sync with one another, wound healing concludes with minimal scar formation. However, the initial mucosal injury in iLTS is thought to develop into a chronic foreign body inflammatory reaction mediated by prolonged and aberrant wound healing pathways. When ECM production exceeds remodeling by MMPs, the net accumulation of ECM leads to scar development and subsequent airway narrowing.

The airway consists of three primary cell types: immune cells, fibroblasts, and epithelial cells. In this review, we will focus on the role of fibroblasts and immune cells and their interactions through inflammatory cytokines that contribute to the development and propagation of iLTS. Specifically, we will discuss the inflammatory pathways mediated by fibroblasts, T-cells, and macrophages that all contribute to this pathologic ECM deposition, as well as potential therapeutics under development to target these pathways in the treatment of iLTS.

\section{Fibroblasts}

Fibroblasts are the key effector cells responsible for the pathologic deposition of collagen that leads to airway narrowing in iLTS. Fibroblasts also have a role in inflammatory signaling and propagating the immune response. Fibroblasts are mesenchymal cells that are not terminally differentiated (14). During homeostasis, fibroblasts are present in the subepithelial tissues in a relatively quiescent state, primarily providing biomechanical support (15). However, in response to injury, fibroblasts are activated and recruited by inflammatory cytokines to proliferate and differentiate into the highly active ECM-secreting myofibroblast phenotype. Fibroblasts are responsible for the production of all ECM components, however in fibroinflammatory diseases there appears to be a relative increase in the production of collagen. In addition, rather than merely responding to inflammatory cytokines to increase production of ECM, fibroblasts actively contribute to the pro-fibrotic mileu through secretion of additional pro-inflammatory cytokines in a positive self-feedback loop that activates additional immune cells. Therefore fibroblasts can be viewed both as end effectors of fibrosis in response to inflammation, and as inflammatory mediators that help shape the immune response to injury $(14,15)$.

Initial investigations into the pathogenesis of iLTS focused on identifying differences between iLTS-derived fibroblasts and normal airway fibroblasts (NAFs). In a rabbit model of iLTS, iLTS-derived fibroblasts were found to possess higher rates of migration and lower contractility than NAFs (16). In addition, comparisons of iLTS scarderived fibroblasts and normal fibroblasts derived from uninjured trachea in the same patients demonstrated significant phenotypic derangements (17). iLTS-derived fibroblasts exhibited increased proliferative capacity, cellular surface area, and expression of collagen-1 and collagen-3 compared to matched normal fibroblasts. Interestingly, the increased collagen expression was not accompanied by an increase in metalloproteinase 2 (MMP2) expression, suggesting disruption in the physiologic balance between collagen production by fibroblasts and its breakdown by MMP2. Metabolic analysis also demonstrated a reduction in oxidative phosphorylation and an increase in the ratio of glycolysis to oxidative phosphorylation in comparison with normal fibroblasts. A similar preference for energy generation via glycolysis rather than oxidative phosphorylation has been observed in rapidly proliferating cancer cells and is termed the Warburg effect. Although oxidative phosphorylation is more efficient in the generation of ATP, glycolysis is more efficient in the conservation of carbon atoms, which are essential for rapidly proliferating cells.

\section{Cytokines}

A variety of cytokines have been described to contribute to the activation and proliferation of fibroblasts leading to the development of fibrosis. Among others, interleukin (IL)-1, IL-6, IL-13, tumor necrosis factor $\alpha$ (TNF- $\alpha)$, transforming growth factor $\beta$ (TGF- $\beta$ ), fibroblast growth factor (FGF), and platelet-derived growth factor (PDGF) promote the profibrotic myofibroblast phenotype that has been observed in iLTS (14). In particular, TGF- $\beta$ and IL-6 have been studied in iLTS and will be discussed further in this section. 
TGF- $\beta$ is a well-described growth factor that is produced by fibroblasts, macrophages and platelets, and has been implicated in fibrosis and scarring in a wide variety of organ systems (18). TGF- $\beta$ directly activates fibroblasts in an autocrine cycle to both further increase production of TGF- $\beta$ and the secretion of ECM proteins (14). Human and animal studies have also observed elevated levels of TGF- $\beta$ in iLTS compared to normal tracheal tissues $(19,20)$. In a rat model of tracheal injury, TGF- $\beta$ expression was found to increase significantly in the first 24-48 hours after injury, then normalized after 21 days (21). When the wounds were exposed to a continuous infusion of TGF- $\beta$, histologic samples demonstrated a notable increase in fibrosis and inflammation, and immunohistochemistry showed increased expression of fibronectin and type I procollagen compared to wounds treated with a saline infusion. This data demonstrates the functional role of TGF- $\beta$ in contributing to fibrosis in iLTS, and identifies it as a potential therapeutic target.

IL-6 is a pro-fibrotic cytokine that has also been implicated in idiopathic pulmonary fibrosis and keloid formation $(22,23)$. It is produced by a variety of cell types, including T-cells, macrophages, skeletal muscle cells, and fibroblasts, acting in both an autocrine and paracrine manner to promote fibrosis (14). A comparison of gene expression from brush biopsies taken from scar and normal trachea in iLTS patients demonstrated a significant increase in the expression of interleukin-6 (IL-6), as well as the myofibroblast marker alpha smooth muscle actin ( $\alpha \mathrm{SMA}$ ), and collagen-1 in scar (24).

Hypoxia has also been shown to play a role in the pathogenesis of iLTS. Tissue ischemia related to high endotracheal tube cuff pressures is thought to contribute to the generation of iLTS (24). When cultured ex vivo under hypoxic conditions, healthy trachea-derived fibroblasts proliferated significantly faster than those cultured in a normoxic environment (24). Culture under hypoxic conditions also induced a significant increase in expression of IL-6 after 6 hours, followed by increased expression of aSMA, collagen-1, and MMP13 after 48 hours. This data provides a temporal correlation suggesting that hypoxia stimulates normal fibroblasts to develop the myofibroblast phenotype of iLTS-derived fibroblasts through the IL-6 pathway.

\section{T-cells}

In contrast to acute inflammatory reactions characterized primarily by neutrophils, chronic inflammation leads to infiltration of T-cells, macrophages, eosinophils and plasma cells. When T-cells are chronically stimulated by contact with antigen, they produce cytokines that activate macrophages, which further activate T-cells leading to a self-perpetuating cycle resulting in fibrosis.

The role of adaptive immunity in the pathogenesis of iLTS was initially demonstrated in a mouse model of iLTS (25). In these experiments designed by Mirza et al., laryngotracheal complexes (LTCs) were harvested from wild type mice and injured mechanically, chemically or left uninjured (25). One of each of these LTCs was implanted into the deep dorsal subcutaneous pockets of wild type and severe combined immunodeficiency (SCID) mice. After three weeks, tissue sections were examined pathologically, and significant granulation tissue was observed in the wild type mice whereas no granulation tissue was observed in the SCID mice. This study demonstrated that the formation of granulation tissue in a mouse model of iLTS is dependent upon systemic circulating B- and/or T-cell mediated processes, because in the absence of these lymphocytes airway injury does not lead to the formation of granulation tissue which is characteristic of iLTS.

Subsequent studies have identified CD4+ helper T-cells as a key mediator of the inflammatory response in iLTS. In general, Th1 responses mediated by interferon- $\gamma$ (IFN- $\gamma$ ) are often described as "pro-inflammatory", while Th2 responses mediated by interleukin-4 (IL-4), IL-5, and IL13 are typically described as "anti-inflammatory" (13). However, when it comes to wound healing, the balance between Th1 and Th2 responses is more nuanced. The Th2 response promotes collagen deposition and ECM remodeling, whereas the Th1 response inhibits ECM production. The balance between Th1 and Th2 responses is thought to protect the body from excessive inflammatory damage in response to an immunologic stimulus. The initial response triggers the Th1 pathway which acts quickly and then subsides with the stimulus without causing further injury. If, however, the immunologic stimulus persists (such as an endotracheal tube causing a persistent foreign body reaction), Th2 and other regulatory cells suppress the Th1 response and promote wound healing in order to prevent excessive tissue damage by a sustained Th1 response (13).

A comparison of brush biopsies from iLTS scar and normal trachea from the same patients identified a significant elevation in IL-4 expression in areas of scar (1). IL-4 is a pro-fibrotic cytokine produced as part of the Th2 response. Elevated IL-4 levels have been observed in 


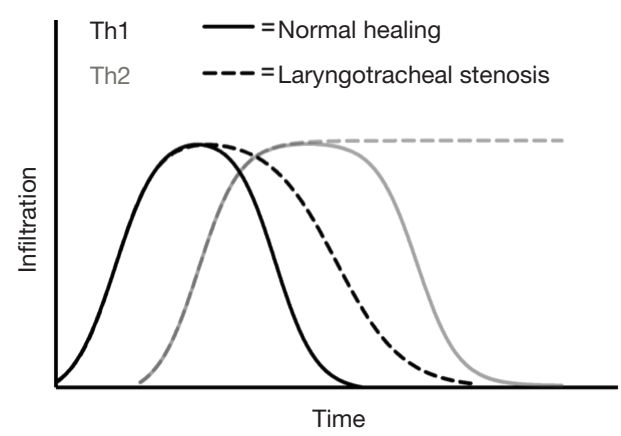

Figure 2 Schematic model of the timing of Th1 and Th2 cell infiltration in normal wound healing compared to LTS. In normal wounds, Th1 infiltration occurs early and subsides rapidly in response to declining immunologic stimulus. In iLTS however, both Th1 and Th2 responses appear to persist beyond a physiologic level, with a predominance of Th2 infiltration in the chronic phase $(1,9,13,27,29)$.

fibrotic diseases such as idiopathic pulmonary fibrosis and have been shown to promote myofibroblast differentiation and proliferation, as well as collagen deposition. A study comparing iLTS specimens to tracheal specimens from normal controls demonstrated significantly higher levels of CD4+ T-cells just deep to the epithelium, in addition to elevated RNA and protein expression of IL-4 (9). In a murine model of iLTS, time course experiments revealed dense CD4+ T-cell infiltration on day 4-7 day after injury, which precedes the development of fibrosis at day 14-21 in the same model $(9,26)$.

These studies demonstrate the role of the Th2 response in mediating development of iLTS. The Th1 response, on the other hand, inhibits fibrosis in a variety of organ systems (13). In patients with iLTS, the Th1 cytokine IFN- $\gamma$ has been shown to inhibit the activity of iLTSderived fibroblasts $e x$ vivo (27). In these experiments, IFN- $\gamma$ treatment suppressed the proliferation and pro-fibrotic function of human iLTS-derived fibroblasts as measured through decreased production of collagen and expression of TGF- $\beta$ (27). Interestingly, increased expression of Th1 cytokines IFN- $\gamma$ and TNF- $\alpha$ have also been observed in a small cohort consisting of nine iLTS patients and one patient with autoimmune LTS, as well as in patients with idiopathic LTS $(1,28)$. This data suggests that strategies aimed at increasing this Th1 response may inhibit fibrosis in iLTS, however further investigation is required to better understand the interplay between these two opposing T-cell phenotypes. Figure 2 provides a schematic representation of our current understanding of the relationship between Th1 and Th2 cell infiltration during normal wound healing and iLTS.

In idiopathic LTS, IL-17 production by delta-gamma T-cells has been implicated in the development of tracheal scar (12). IL-17 production by Th17 cells has also been identified as an additional pro-fibrotic signal in a variety of conditions including idiopathic pulmonary fibrosis, hepatic, and myocardial fibrosis (30). Elevated levels of IL-6 in patients with iLTS coupled with the induction of IL-6 under hypoxic conditions provides a theoretical stimulus for Th17 cell differentiation in iLTS (24), however significant differences in IL-17 expression have not been observed in iLTS. Whether this is due to a lack of power in currently published studies, or a non-contributive role of IL-17 in the pathogenesis of iLTS remains to be seen in future investigations.

\section{Macrophages}

In addition to T-cells, macrophages represent another immune effector cell that plays a central role in regulating the inflammatory response and has been implicated in the pathogenesis of iLTS. Macrophages contribute to normal wound-healing through phagocytosis of cellular debris, stimulation of T-cells and fibroproliferation through cytokine signaling. Dysregulated macrophage function has been associated with excessive fibrosis in a variety of organ systems (13). IFN- $\gamma$ produced by Th1 cells induces nitricoxide synthetase 2 (NOS2) in "classically activated" macrophages (CAM or M1 macrophages), thereby decreasing collagen synthesis by fibroblasts (13). However, IL-4 and IL-13 produced by Th2 cells stimulate arginase-1 (Arg1) activity in "alternatively activated" (M2) macrophages, thereby promoting fibroblast proliferation and collagen production. Given the previously described Th2 response seen in iLTS patients, studies have turned to evaluating the role of M2 macrophages in the pathogenesis of iLTS.

In vitro co-culture studies of vocal fold scar-derived fibroblasts and monocytes demonstrated induction of an M2 phenotype by scar-derived fibroblasts with a decrease in production of M1 cytokines TNF-a and IL-12 and an increase in M2 cytokine IL-10 (31). In a chemomechanical injury murine model of iLTS, initial M1 macrophage response mirrored that seen in mechanical injury controls that represent normal wound healing (29). However by day 10 after injury the chemomechanical iLTS mice exhibited higher levels of M2 macrophage infiltration 
compared to controls (29). Gene expression of macrophage marker CD11b and the acute inflammatory cytokine IL-I $\beta$ increased on day 1 after chemomechanical injury compared to mechanical injury and mock surgery controls. These changes were followed by a decrease in expression of M1 marker inducible nitric oxide synthetase (iNOS) and an increase in expression of M2 marker Arg1 on day 7 after injury. This shift in macrophage phenotype on day 7 was also associated with an increase in expression of collagen I, TGF , and elastin. By day 10 after injury, flow cytometry demonstrated an increase in M1 macrophage content of scar compared to mock controls and an increase in M2 macrophage content compared to both mechanical injury and mock controls (29). A study of nine patients with iLTS and one with autoimmune LTS identified elevated levels of the macrophage growth factor granulocyte macrophage colony-stimulating factor (GM-CSF), in addition to the M2 cytokine IL-10 when compared to controls (28). Taken together, this animal and human data indicates that M2 macrophages play a significant role in the pathogenesis of iLTS, and are likely stimulated by both the Th2 response as well as fibroblasts, themselves.

\section{Therapeutic approaches}

Current options for medical adjuncts to the surgical treatment of iLTS include steroids, mitomycin C, antibiotics and other anti-inflammatory medications. Unfortunately, none of these therapies has been consistently shown to improve outcomes for iLTS patients, and repeated surgical procedures remains the mainstay of management for most patients. However, given the advances underway in our understanding of the fibroinflammatory mechanisms of iLTS, potential is rising for the development of targeted immunologic therapies for the treatment of iLTS. In this section we will discuss novel therapeutic approaches under preclinical investigation to target inflammation in iLTS.

\section{Rapamycin}

Rapamycin is an FDA-approved macrocyclic antibiotic with immunosuppressive and antiproliferative functions that is used as an anti-rejection agent in solid organ transplants (32). Rapamycin is produced by the bacteria streptomyces hygroscopicus, and binds with FK-binding protein 12 to inhibit the mammalian target of rapamycin (mTOR). mTOR regulates cell cycle progression and extracellular matrix production in fibroblasts, as well as lymphocyte proliferation and activation. Therefore, inhibition of mTOR by rapamycin has been studied in fibrosis of a variety of organ systems to both directly target fibroblast function and to suppress the lymphocytes that stimulate them (33).

In vitro studies of human iLTS-derived fibroblasts treated with rapamycin demonstrated a significant decrease in fibroblast proliferation and Collagen I expression compared to iLTS-derived fibroblasts treated with vehicle control (33). In addition, rapamycin reduced ATP production and maximal and basal respiration rates suggesting that a reduction in oxidative phosphorylation is at least in part responsible for the rapamycin-mediated decrease in fibroblast activity. In vivo studies of a rapamycin-eluting stent in a murine model of iLTS found a significant reduction in the thickness of the lamina propria and in expression of collagen 3 , TGF- $\beta$, and $\alpha$ SMA (34). These experiments demonstrate the ability of a rapamycineluting stent to reduce stenosis in iLTS through decreased fibroblast proliferation, deposition of collagen, and myofibroblast differentiation.

\section{Anti-TGF- $\beta$}

TGF- $\beta$ has been implicated in a wide variety of fibrotic diseases, and as seen in other fibroinflammatory conditions, iLTS patients exhibit elevated levels of TGF- $\beta$ in diseased tissue $(19,20)$. A number of anti-TGF- $\beta$ small molecule inhibitors and humanized monoclonal antibodies have been developed, and are undergoing clinical investigation in cancer and idiopathic pulmonary fibrosis (35). However, although these agents have shown promise in preclinical studies, they have yet to be evaluated in patients with iLTS.

These preclinical studies include evaluation of direct wound infusions with anti-TGF- $\beta$ in a rat model of tracheal injury. In this study, anti-TGF- $\beta$ infusion induced a decrease in fibronectin and type I procollagen compared to controls, demonstrating the reversibility of the TGF- $\beta$-mediated effects (21). Additionally, in a cautery-induced canine model of iLTS, a combination of intralesional and intravenous injections of anti-TGF- $\beta$ significantly reduced the degree of tracheal stenosis and increased survival time compared to treatment with saline control (36).

\section{Additional areas of investigation}

IL-17 is another potential target for the treatment of idiopathic LTS. Therapeutics targeting the IL-17 pathway 


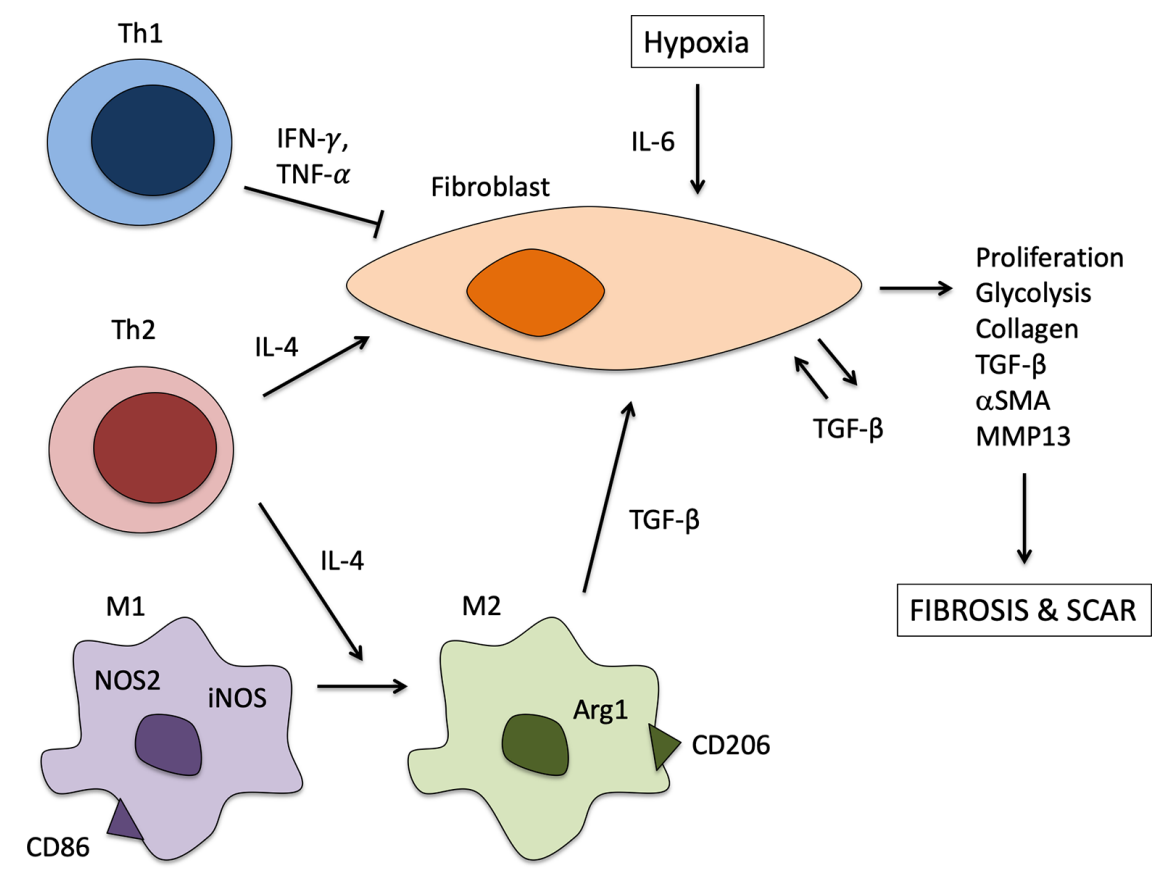

Figure 3 Summary of the Th2/M2 response in iLTS. IL-4 secretion by Th2 cells activates fibroblasts to proliferate, produce collagen, and acquire a myofibroblast phenotype. IL-4 also stimulates M2 differentiation, and M2 macrophages then secrete TGF- $\beta$ to further stimulate fibroblasts. Activated fibroblasts also produce TGF- $\beta$ in a positive feedback loop to further stimulate fibrosis.

have been developed, and are FDA approved for the treatment of psoriasis and ankylosing spondylitis. While a few rheumatologists have prescribed anti-IL-17 therapy for idiopathic subglottic stenosis patients, the drug's efficacy has not been determined at the time of this review.

The immunosuppressive agent, tacrolimus, is also under investigation for the treatment of iLTS. Like rapamycin, tacrolimus is FDA approved as an anti-rejection agent in recipients of solid organ transplants. Tacrolimus is a calcineurin inhibitor that prevents T-cell activation, thereby possessing both antiproliferative and anti-inflammatory activities (37). In a rat model of iLTS, low-dose systemic tacrolimus was found to decrease the degree of airway stenosis through inhibition of the calcineurin/nuclear factor of activated T-cell/IL-2 pathway (37). A study of airway stent-induced granulation tissue in transplant recipients receiving an immunosuppressive regimen including tacrolimus also demonstrated a reduction in airway narrowing (38), however tacrolimus has yet to be evaluated independently in the treatment of patients with iLTS.

\section{Conclusions}

Although there is still much to learn about the detailed roles of various inflammatory pathways in the pathogenesis of iLTS, the paradigm shift in understanding iLTS as a fibroinflammatory disease has led to the identification of the Th2/M2 response as a critical piece of the puzzle (Figure 3). Both human studies and animal models show increased presence of subepithelial Th2 cells as well as Th2 cytokines in iLTS scar. A shift from the M1 to the M2 macrophage phenotype has also been observed over time, suggesting that in addition to directly stimulating fibroblasts to increase collagen deposition, Th2 cells also contribute to M2 macrophage differentiation and their pro-fibrotic functions. Two therapeutic approaches targeting inflammation in iLTS are under investigation, including rapamycin-eluting stents and anti-TGF- $\beta$ antibodies, which have both shown promise in their ability to decrease airway narrowing and collagen deposition in animal models of iLTS. Further understanding of the inflammatory mechanisms contributing to fibrosis in iLTS will generate targets for mechanistically-directed therapies with the goal of improving outcomes for this chronic and potentially life-threatening condition.

\section{Acknowledgments}

Funding: Research reported in his publication was 
supported by National Institute on Deafness and Other Communication Disorders of the National Institutes of Health under award number 1K23DC014082 and R21DC017225. The content is solely the responsibility of the authors and does not necessarily represent the official views of the National Institutes of Health. This study was also financially supported by the Tribological Society and American College of Surgeons.

\section{Footnote}

Provenance and Peer Review: This article was commissioned by the Guest Editors (Benoit Jacques Bibas, Paulo Francisco Guerreiro Cardoso and Konrad Hoetzenecker) for the series "Recent Developments in Benign Tracheal Stenosis" published in Translational Cancer Research. The article has undergone external peer review.

Conflicts of Interest: Both authors have completed the ICMJE uniform disclosure form (available at http:// dx.doi.org/10.21037/tcr.2020.01.21). The series "Recent Developments in Benign Tracheal Stenosis" was commissioned by the editorial office without any funding or sponsorship. ATH reports grants from NIH/NIDCD, during the conduct of the study; grants from Medtronic, personal fees from Ambu, USA, personal fees from Olympus, USA, outside the submitted work. The authors have no other conflicts of interest to declare.

Ethical Statement: The authors are accountable for all aspects of the work in ensuring that questions related to the accuracy or integrity of any part of the work are appropriately investigated and resolved.

Open Access Statement: This is an Open Access article distributed in accordance with the Creative Commons Attribution-NonCommercial-NoDerivs 4.0 International License (CC BY-NC-ND 4.0), which permits the noncommercial replication and distribution of the article with the strict proviso that no changes or edits are made and the original work is properly cited (including links to both the formal publication through the relevant DOI and the license). See: https://creativecommons.org/licenses/by-nc-nd/4.0/.

\section{References}

1. Motz KM, Yin LX, Samad I, et al. Quantification of Inflammatory Markers in Laryngotracheal Stenosis.
Otolaryngol Head Neck Surg 2017;157:466-72.

2. Gelbard A, Francis DO, Sandulache VC, et al. Causes and consequences of adult laryngotracheal stenosis. Laryngoscope 2015;125:1137-43.

3. Manica D, Schweiger C, Maróstica PJC, et al. Association Between Length of Intubation and Subglottic Stenosis in Children. Laryngoscope 2013;123:1049-54.

4. Hillel AT, Karatayli-Ozgursoy S, Benke JR, et al. Voice quality in laryngotracheal stenosis: impact of dilation and level of stenosis. Ann Otol Rhinol Laryngol 2015;124:413-8.

5. Yin LX, Padula W V., Gadkaree S, et al. Health Care Costs and Cost-effectiveness in Laryngotracheal Stenosis. Otolaryngol Head Neck Surg 2019;160:679-86.

6. Hseu AF, Benninger MS, Haffey TM, et al. Subglottic stenosis: a ten-year review of treatment outcomes. Laryngoscope 2014;124:736-41.

7. Hirshoren N, Eliashar R. Wound-healing modulation in upper airway stenosis-Myths and facts. Head Neck 2009;31:111-26.

8. Minnigerode B, Richter HG. Pathophysiology of subglottic tracheal stenosis in childhood. Prog Pediatr Surg 1987;21:1-7.

9. Hillel AT, Ding D, Samad I, et al. T-Helper 2 Lymphocyte Immunophenotype Is Associated With Iatrogenic Laryngotracheal Stenosis. Laryngoscope 2019;129:177-86.

10. Dohar JE, Klein EC, Betsch JL, et al. Fetal airway wound repair: a new frontier. Arch Otolaryngol Head Neck Surg 1998;124:25-9.

11. Branski RC, Sandulache VC, Dohar JE, et al. Mucosal wound healing in a rabbit model of subglottic stenosis: biochemical analysis of secretions. Arch Otolaryngol Head Neck Surg 2005;131:153-7.

12. Gelbard A, Katsantonis N-G, Mizuta M, et al. Idiopathic subglottic stenosis is associated with activation of the inflammatory IL-17A/IL-23 axis. Laryngoscope 2016;126:E356-61.

13. Wynn TA. Fibrotic disease and the TH1/TH2 paradigm. Nat Rev Immunol 2004;4:583-94.

14. Kendall RT, Feghali-Bostwick CA. Fibroblasts in fibrosis: novel roles and mediators. Front Pharmacol 2014;5:123.

15. Morrison RJ, Katsantonis NG, Motz KM, et al. Pathologic Fibroblasts in Idiopathic Subglottic Stenosis Amplify Local Inflammatory Signals. Otolaryngol Head Neck Surg 2019;160:107-15.

16. Singh T, Sandulache VC, Otteson TD, et al. Subglottic stenosis examined as a fibrotic response to airway injury characterized by altered mucosal fibroblast activity. Arch 
Otolaryngol Head Neck Surg 2010;136:163-70.

17. Ma G, Samad I, Motz K, et al. Metabolic variations in normal and fibrotic human laryngotracheal-derived fibroblasts: a Warburg-like effect. Laryngoscope 2017;127:E107-13.

18. Biernacka A, Dobaczewski M, Frangogiannis NG. TGF- $\beta$ signaling in fibrosis. Growth Factors 2011;29:196-202.

19. Scioscia KA, Miller F, April MM, et al. Growth factors in subglottic stenosis. Ann Otol Rhinol Laryngol 1996;105:936-43.

20. Karagiannidis C, Velehorschi V, Obertrifter B, et al. High-level expression of matrix-associated transforming growth factor-beta 1 in benign airway stenosis. Chest 2006;129:1298-304.

21. Dillard DG, Gal AA, Roman-Rodriguez J, et al. Transforming growth factor and neutralizing antibodies in subglottic stenosis. Ann Otol Rhinol Laryngol 2001;110:393-400.

22. Ghazizadeh M, Tosa M, Shimizu H, et al. Functional implications of the IL-6 signaling pathway in keloid pathogenesis. J Invest Dermatol 2007;127:98-105.

23. Le TTT, Karmouty-Quintana H, Melicoff E, et al. Blockade of IL-6 Trans signaling attenuates pulmonary fibrosis. J Immunol 2014;193:3755-68.

24. Yin LX, Motz KM, Samad I, et al. Fibroblasts in Hypoxic Conditions Mimic Laryngotracheal Stenosis. Otolaryngol Head Neck Surg 2017;156:886-892.

25. Ghosh A, Malaisrie N, Leahy KP, et al. Cellular Adaptive Inflammation Mediates Airway Granulation in a Murine Model of Subglottic Stenosis. Otolaryngol Head Neck Surg 2011;144:927-33.

26. Hillel AT, Namba D, Ding D, et al. An in situ, in vivo murine model for the study of laryngotracheal stenosis. JAMA Otolaryngol Head Neck Surg 2014;140:961-6.

27. Motz K, Samad I, Yin LX, et al. Interferon- $\gamma$ Treatment of Human Laryngotracheal Stenosis-Derived Fibroblasts.

Cite this article as: Davis RJ, Hillel AT. Inflammatory pathways in the pathogenesis of iatrogenic laryngotracheal stenosis: what do we know? Transl Cancer Res 2020;9(3):21082116. doi: 10.21037/tcr.2020.01.21
JAMA Otolaryngol Head Neck Surg 2017;143:1134-40.

28. Haft S, Lee JY, Ghosh A, et al. Inflammatory Protein Expression in Human Subglottic Stenosis Tissue Mirrors That in a Murine Model. Ann Otol Rhinol Laryngol 2014;123:65-70.

29. Hillel AT, Samad I, Ma G, et al. Dysregulated Macrophages Are Present in Bleomycin-Induced Murine Laryngotracheal Stenosis. Otolaryngol Head Neck Surg 2015;153:244-50.

30. Wynn TA, Ramalingam TR. Mechanisms of fibrosis: therapeutic translation for fibrotic disease. Nat Med 2012;18:1028-40.

31. King SN, Chen F, Jetté ME, et al. Vocal fold fibroblasts immunoregulate activated macrophage phenotype. Cytokine 2013;61:228-36.

32. Hillel AT, Gelbard A. Unleashing rapamycin in fibrosis. Oncotarget 2015;6:15722-3.

33. Namba DR, Ma G, Samad I, et al. Rapamycin Inhibits Human Laryngotracheal Stenosis-derived Fibroblast Proliferation, Metabolism, and Function in Vitro. Otolaryngol Head Neck Surg 2015;152:881-8.

34. Duvvuri M, Motz K, Murphy M, et al. Engineering an immunomodulatory drug-eluting stent to treat laryngotracheal stenosis. Biomater Sci 2019;7:1863-74.

35. de Gramont A, Faivre S, Raymond E. Novel TGF- $\beta$ inhibitors ready for prime time in onco-immunology. Oncoimmunology 2016;6:e1257453.

36. Simpson CB, White S, McGuff HS. Anti-Transforming Growth Factor Beta as a Treatment for Laryngotracheal Stenosis in a Canine Model. Laryngoscope 2008;118:546-51.

37. Mizokami D, Araki K, Tanaka N, et al. Tacrolimus prevents laryngotracheal stenosis in an acute-injury rat model. Laryngoscope 2015;125:E210-5.

38. Shlomi D, Peled N, Shitrit D, et al. Protective effect of immunosuppression on granulation tissue formation in metallic airway stents. Laryngoscope 2008;118:1383-8. 